\title{
Comparison between air pollution
}

\section{concentrations measured at the nearest} monitoring station to the delivery hospital and those measured at stations nearest the residential postal code regions of pregnant women in Fukuoka

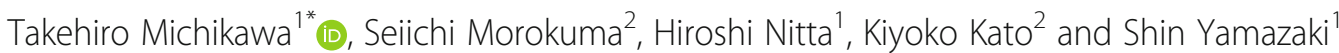

\begin{abstract}
Background: Numerous earlier studies examining the association of air pollution with maternal and foetal health estimated maternal exposure to air pollutants based on the women's residential addresses. However, residential addresses, which are personally identifiable information, are not always obtainable. Since a majority of pregnant women reside near their delivery hospitals, the concentrations of air pollutants at the respective delivery hospitals may be surrogate markers of pollutant exposure at home. We compared air pollutant concentrations measured at the nearest monitoring station to Kyushu University Hospital with those measured at the closest monitoring stations to the respective residential postal code regions of pregnant women in Fukuoka.

Methods: Aggregated postal code data for the home addresses of pregnant women who delivered at Kyushu University Hospital in 2014 was obtained from Kyushu University Hospital. For each of the study's 695 women who resided in Fukuoka Prefecture, we assigned pollutant concentrations measured at the nearest monitoring station to Kyushu University Hospital and pollutant concentrations measured at the nearest monitoring station to their respective residential postal code regions.

Results: Among the 695 women, 584 (84.0\%) resided in the proximity of the nearest monitoring station to hospital or one of the four other stations (as the nearest stations to their respective residential postal code region) in Fukuoka city. Pearson's correlation for daily mean concentrations among the monitoring stations in Fukuoka city was strong for fine particulate matter ( $P M_{2.5}$ ), suspended particulate matter (SPM), and photochemical oxidants (Ox) (coefficients $\geq 0.9$ ), but moderate for coarse particulate matter (the result of subtracting the $\mathrm{PM}_{2.5}$ from the SPM concentrations), nitrogen dioxide, and sulphur dioxide. Hospital-based and residence-based concentrations of PM 2.5 , SPM, and Ox were comparable.
\end{abstract}

Conclusions: For PM 2.5 , SPM, and Ox, exposure estimation based on the delivery hospital is likely to approximate that based on the home of pregnant women.

Keywords: Air pollution, Monitoring station, Pregnant woman, Validation

\footnotetext{
* Correspondence: tmichikawa@nies.go.jp

${ }^{1}$ Environmental Epidemiology Section, Centre for Health and Environmental

Risk Research, National Institute for Environmental Studies, 16-2 Onogawa,

Tsukuba, Ibaraki 305-8506, Japan

Full list of author information is available at the end of the article
} 


\section{Background}

There is increasing evidence for an association between air pollution and maternal and foetal health. Since the health effects of air pollution are relatively small, a large sample size is required in order to investigate this association. Therefore, numerous earlier studies used registry data to increase the number of the participants [1-7]. In such a study design, however, personal exposure to respirable pollutants could not be measured. Thus, individual exposure to air pollutants at the participants' home addresses has been estimated based on concentrations measured at nearby ambient air monitoring stations or on a statistical model similar to land use regression models [3-7].

Our research question is whether recent air pollution in Japan influences maternal and foetal health. In Japan, however, there is no nationwide public registry data that includes sufficient information on outcome and confounders related to maternal and foetal health. Accumulated data regarding maternal and foetal health may be available if we collaborate with obstetric health care providers. Even in this case, however, we cannot collect relevant personally identifiable information, (i.e. residential addresses) to estimate maternal exposure to air pollutants, without informed consent from the subjects [8], and it is not easy to receive such informed consent from women who have given birth and completed the obstetric follow-up process. However, we may have access to anonymised information including relevant data on pregnant women who delivered at cooperating hospitals. Although anonymised data does not allow us to estimate residence-based maternal exposure to pollutants, a majority of pregnant women reside near their delivery hospitals in Japan $[9,10]$. Based on the assumption, then, that pregnant women reside near their delivery hospital, we may be able to use the air pollutant concentrations at the respective delivery hospitals as surrogate markers of pollutant exposure at their homes.

Our a priori hypothesis is that pollutant exposure estimation based on the delivery hospital approximates exposure estimation based on the home address of pregnant women. To examine this hypothesis, we used aggregated residential postal code data for pregnant women who delivered at Kyushu University Hospital and compared the air pollutant concentrations measured at the nearest monitoring station to Kyushu University Hospital with those measured at the nearest monitoring stations to the respective residential postal code regions of the women.

\section{Materials and methods}

\section{Study data}

Aggregated postal code data for the home addresses of pregnant women who delivered at Kyushu University Hospital in 2014 was obtained from Kyushu University
Hospital, Higashi-ku, Fukuoka city, Fukuoka Prefecture. As we only collected aggregated data (e.g. the number of women who resided in the region of postal code 8120041 was 17), we did not have individual information, such as maternal age and delivery date. In 2014, a total of 784 women gave birth in Kyushu University Hospital. Among them, we excluded 89 women who resided outside Fukuoka Prefecture because they would have returned to parental homes (according to the Japanese satogaeri custom) near the hospital and included in this study 695 women who resided in Fukuoka Prefecture. The study protocol was approved by the Institutional Review Board of Kyushu University, Japan, and the Japan National Institute for Environmental Studies.

\section{Environmental data}

We used daily mean concentrations of fine particulate matter $\left(\mathrm{PM}_{2.5}\right.$, airborne particles with a $50 \%$ cut-off level at $2.5-\mu \mathrm{m}$ aerodynamic diameter) measured by $\beta$-ray attenuation method, suspended particulate matter (SPM, airborne particles with a $100 \%$ cut-off level at $10-\mu \mathrm{m}$ aerodynamic diameter) measured by $\beta$-ray attenuation method, nitrogen dioxide $\left(\mathrm{NO}_{2}\right)$ measured by colorimetry employing Saltzman reagent or chemiluminescent method using ozone, and sulphur dioxide $\left(\mathrm{SO}_{2}\right)$ measured by conductometry or ultraviolet fluorescence method, and maximum 8-h mean concentrations of photochemical oxidants $(\mathrm{Ox})$ measured by ultraviolet absorption spectrometry. These concentrations were measured at the ambient air monitoring stations representing background pollutant concentrations in the respective areas and were stored in the atmospheric environment database of the Japan National Institute for Environmental Studies. The same measurement method for each of $\mathrm{NO}_{2}$ and $\mathrm{SO}_{2}$ (chemiluminescent method for $\mathrm{NO}_{2}$ and ultraviolet fluorescence method for $\mathrm{SO}_{2}$ ) was used in the monitoring stations within Fukuoka city. In this study, the concentration of coarse particulate matter (coarse PM) was defined as the result of subtracting the $\mathrm{PM}_{2.5}$ concentration from the SPM concentration.

We converted the aggregated data into individual data and randomly assigned, to each woman, 1 day in 2014 as a delivery day. We linked the individual delivery days and pollutant concentrations at the nearest monitoring station measuring $\mathrm{PM}_{2.5}$ to the respective residential postal code regions of the participants. The centroid of each region was used to measure the linear distance from the nearest monitoring station. In addition, we assigned pollutant concentrations measured at the nearest monitoring station to Kyushu University Hospital (the Yoshizuka monitoring station, approximately $1 \mathrm{~km}$ east of the hospital) to all participants. The hospital and monitoring station locations are shown in Fig. 1. In the past studies examining the association between air pollution and 


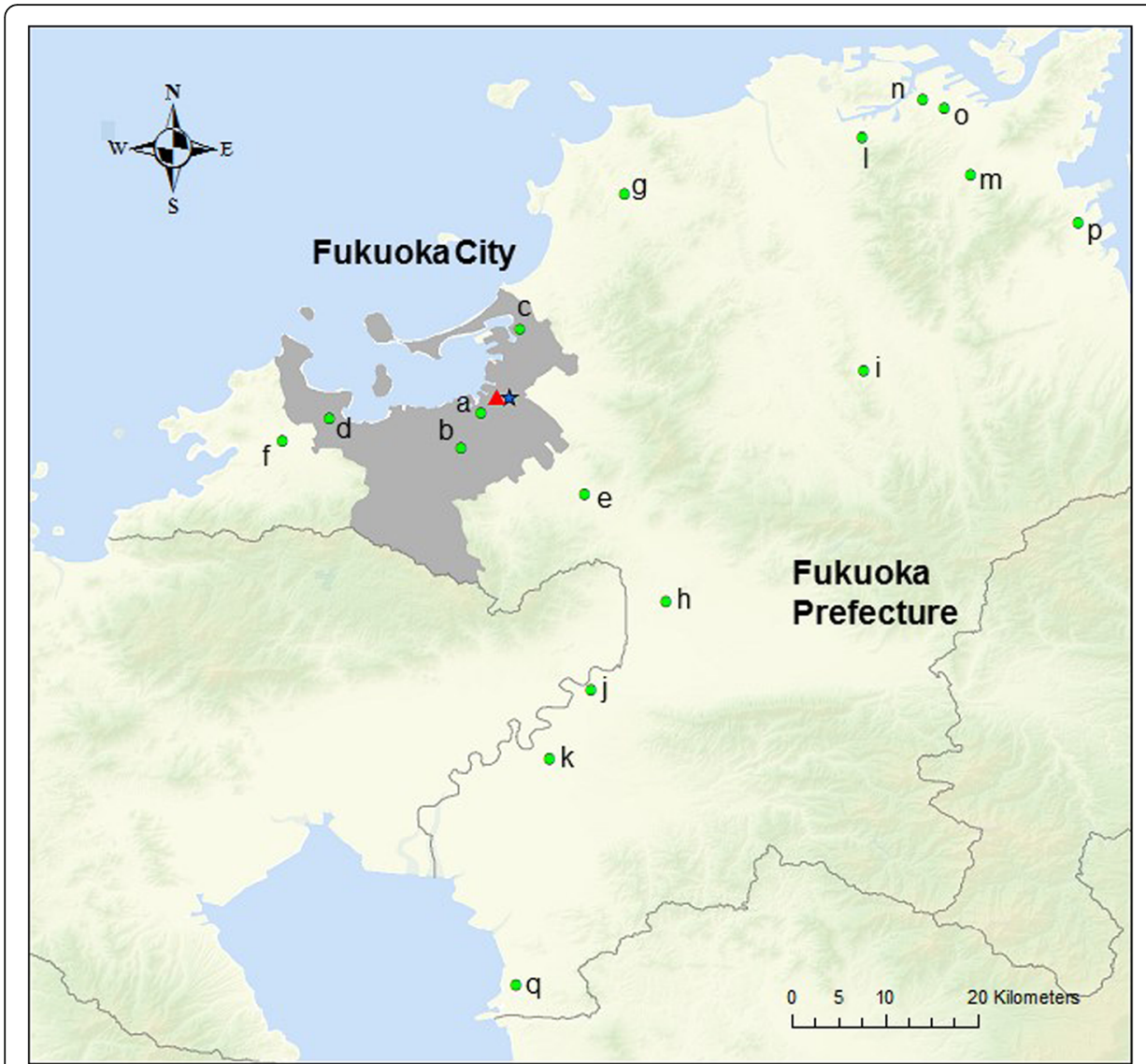

Kyushu University Hospital

Yoshizuka monitoring station

Nearest monitoring station to residential region

Fig. 1 Locations of hospital and monitoring stations

maternal and foetal health, periods of exposure estimate were set at day, week (representing a pregnancy week average), month (representing a pregnancy month average), and 3 months (representing a trimester average) [3, 7, 11-13]. Therefore, concentration values were estimated for the following periods: 1-day average (day of delivery), 7-day average, 1-month average, and 3-month average.

\section{Statistical analysis}

We summarised residential information of study population. Pearson's correlation coefficients of daily mean pollutant concentrations among the monitoring stations were calculated. Percentage differences were defined as the differences between concentrations measured at the nearest monitoring stations to the residential regions and those measured at the Yoshizuka station, divided by the concentrations measured at the Yoshizuka station. All analyses were performed using Stata14 for Windows (Stata Corporation, College Station, TX, USA).

\section{Results}

Among the 695 women who resided in Fukuoka Prefecture, $641(92.2 \%)$ resided within a $20-\mathrm{km}$ radius of the Yoshizuka monitoring station, 584 (84.0\%) resided near the Yoshizuka station or one of the four other monitoring stations (as the nearest stations to their respective residential postal code region) in Fukuoka city, and 279 (40.1\%) resided near the Yoshizuka station (Table 1). In

Table 1 Study population

\begin{tabular}{|c|c|c|}
\hline & Number & Percent \\
\hline Women who delivered at Kyushu University Hospital and resided in Fukuoka Prefecture & 695 & 100 \\
\hline Women who resided within a 20-km radius of the Yoshizuka monitoring station (the nearest station to Kyushu University Hospital) & 641 & 92.2 \\
\hline $\begin{array}{l}\text { Women who resided near Yoshizuka station or one of the four other monitoring stations (as the nearest station to their } \\
\text { respective residential postal code region) in Fukuoka city }\end{array}$ & 584 & 84.0 \\
\hline Women who resided in Fukuoka city & 433 & 62.3 \\
\hline Women who resided near Yoshizuka monitoring station (as the nearest station to their respective residential postal code region) & 279 & 40.1 \\
\hline
\end{tabular}


the case of $99.4 \%$ of the women, the linear distance between their respective residential postal code region and the nearest monitoring station was less than $10 \mathrm{~km}$, while $75.7 \%$ of women resided within a $5-\mathrm{km}$ radius of the monitoring station.

We investigated the correlation between daily mean pollutant concentrations among the monitoring stations in 2014. Among the monitoring stations in Fukuoka city (Yoshizuka and four other monitoring stations), the concentrations of $\mathrm{PM}_{2.5}$ were highly correlated, with Pearson's correlation coefficients ranging from 0.96 to 0.99 (Table 2). The coefficient between $\mathrm{PM}_{2.5}$ concentrations measured at the Yoshizuka station and those at the farthest station from Yoshizuka station (linear distance approximately $65 \mathrm{~km}$ ) was 0.87 . Also, the concentrations of SPM and Ox were well correlated among the monitoring stations in and outside Fukuoka city (Additional file 1: Table S1). The correlation coefficients among the coarse PM concentrations measured at the Yoshizuka station and those measured at other stations ranged from 0.21 to 0.74 . In the case of $\mathrm{NO}_{2}$ and $\mathrm{SO}_{2}$ concentrations, the correlation coefficients among monitoring stations in Fukuoka city, in which the same measurement method for each of $\mathrm{NO}_{2}$ and $\mathrm{SO}_{2}$ was used, ranged from 0.76 to 0.87 , and the smallest coefficients between the Yoshizuka station and another station were 0.46 for $\mathrm{NO}_{2}$ and 0.43 for $\mathrm{SO}_{2}$.
Table 3 shows comparisons between the pollutant concentrations measured at the Yoshizuka monitoring station and those measured at the nearest monitoring stations to the respective residential postal code regions. Mean $\mathrm{PM}_{2.5}$ concentrations at the Yoshizuka station $\left(17.0 \mu \mathrm{g} / \mathrm{m}^{3}\right.$ for 1 day average, $17.7 \mu \mathrm{g} / \mathrm{m}^{3}$ for 7 -day average, $18.0 \mu \mathrm{g} / \mathrm{m}^{3}$ for 1-month average, and $18.1 \mu \mathrm{g} / \mathrm{m}^{3}$ for 3-month average) were similar to those at the nearest stations $\left(17.2 \mu \mathrm{g} / \mathrm{m}^{3}\right.$ for 1-day average, $17.9 \mu \mathrm{g} / \mathrm{m}^{3}$ for 7 -day average, $18.2 \mu \mathrm{g} / \mathrm{m}^{3}$ for 1-month average, and $18.4 \mu \mathrm{g} / \mathrm{m}^{3}$ for 3-month average). Such statistical results were also observed for Ox. The percentage differences for $\mathrm{PM}_{2.5}$ and $\mathrm{Ox}$ ranged from 1.0 to $2.8 \%$. For SPM, the percentage differences were around $6.5 \%$, though the concentrations at the Yoshizuka station tended to be slightly lower than those at the nearest stations. The percentage differences of hospital-based and residence-based coarse $\mathrm{PM}, \mathrm{NO}_{2}$, and $\mathrm{SO}_{2}$ concentrations ranged from 11.3 to $26.1 \%$. Complementally, we show the correlation between pollutant concentrations measured at the Yoshizuka monitoring station and those measured at the nearest monitoring stations to the respective residential postal code regions in Additional file 1: Table S2.

\section{Discussion}

We used the residential postal codes of pregnant women who delivered at Kyushu University Hospital and compared

Table 2 Pearson's correlation coefficients for daily mean fine particulate matter $\left(\mathrm{PM}_{2.5}\right)$ concentrations among monitoring stations in Fukuoka, 2014

\begin{tabular}{|c|c|c|c|c|c|c|c|c|c|c|c|c|c|c|c|c|c|c|}
\hline \multirow{2}{*}{$\begin{array}{l}\text { Monitoring } \\
\text { stationa }^{\mathrm{a}}\end{array}$} & \multicolumn{5}{|c|}{ In Fukuoka city } & \multicolumn{13}{|c|}{ Outside Fukuoka city } \\
\hline & Yoshizuka & $\mathrm{a}$ & $b$ & c & d & $\mathrm{e}$ & $f$ & g & h & i & j & k & I & m & $n$ & o & $p$ & q \\
\hline Yoshizuka & 1 & & & & & & & & & & & & & & & & & \\
\hline a & 0.99 & 1 & & & & & & & & & & & & & & & & \\
\hline b & 0.97 & 0.98 & 1 & & & & & & & & & & & & & & & \\
\hline c & 0.97 & 0.97 & 0.97 & 1 & & & & & & & & & & & & & & \\
\hline$d$ & 0.96 & 0.96 & 0.96 & 0.96 & 1 & & & & & & & & & & & & & \\
\hline e & 0.95 & 0.95 & 0.96 & 0.93 & 0.91 & 1 & & & & & & & & & & & & \\
\hline f & 0.94 & 0.94 & 0.96 & 0.93 & 0.94 & 0.95 & 1 & & & & & & & & & & & \\
\hline g & 0.94 & 0.94 & 0.94 & 0.94 & 0.92 & 0.95 & 0.95 & 1 & & & & & & & & & & \\
\hline h & 0.93 & 0.92 & 0.93 & 0.89 & 0.87 & 0.97 & 0.92 & 0.91 & 1 & & & & & & & & & \\
\hline i & 0.94 & 0.93 & 0.94 & 0.92 & 0.90 & 0.96 & 0.93 & 0.95 & 0.94 & 1 & & & & & & & & \\
\hline j & 0.93 & 0.92 & 0.92 & 0.89 & 0.87 & 0.95 & 0.90 & 0.88 & 0.97 & 0.92 & 1 & & & & & & & \\
\hline k & 0.82 & 0.79 & 0.78 & 0.75 & 0.72 & 0.82 & 0.75 & 0.72 & 0.88 & 0.78 & 0.93 & 1 & & & & & & \\
\hline | & 0.96 & 0.95 & 0.94 & 0.94 & 0.93 & 0.92 & 0.92 & 0.95 & 0.90 & 0.95 & 0.88 & 0.74 & 1 & & & & & \\
\hline $\mathrm{m}$ & 0.92 & 0.91 & 0.91 & 0.91 & 0.89 & 0.91 & 0.90 & 0.94 & 0.89 & 0.95 & 0.87 & 0.72 & 0.96 & 1 & & & & \\
\hline$n$ & 0.93 & 0.93 & 0.92 & 0.93 & 0.92 & 0.89 & 0.90 & 0.93 & 0.86 & 0.93 & 0.84 & 0.68 & 0.97 & 0.96 & 1 & & & \\
\hline o & 0.92 & 0.92 & 0.92 & 0.92 & 0.91 & 0.90 & 0.90 & 0.93 & 0.87 & 0.94 & 0.85 & 0.70 & 0.97 & 0.97 & 0.98 & 1 & & \\
\hline$p$ & 0.88 & 0.88 & 0.87 & 0.88 & 0.86 & 0.89 & 0.87 & 0.92 & 0.87 & 0.93 & 0.82 & 0.65 & 0.92 & 0.97 & 0.93 & 0.94 & 1 & \\
\hline q & 0.87 & 0.87 & 0.87 & 0.82 & 0.80 & 0.91 & 0.86 & 0.84 & 0.93 & 0.89 & 0.94 & 0.89 & 0.84 & 0.83 & 0.79 & 0.80 & 0.79 & 1 \\
\hline
\end{tabular}

${ }^{a}$ The locations of monitoring stations from a to $q$ are shown in Fig. 1. The alphabetical order reflects the distance from the Yoshizuka station (i.e. a is the nearest station and $q$ is the farthest station from the Yoshizuka station) 
Table 3 Comparisons between pollutant concentrations measured at the Yoshizuka monitoring station and those at the nearest monitoring stations to the respective residential postal code regions

\begin{tabular}{|c|c|c|c|c|c|c|c|}
\hline & \multicolumn{3}{|c|}{$\begin{array}{l}\text { Concentrations measured at the nearest monitoring } \\
\text { stations to the respective residential regions }\end{array}$} & \multicolumn{3}{|c|}{$\begin{array}{l}\text { Concentrations measured at the Yoshizuka } \\
\text { monitoring station }\end{array}$} & \multirow{2}{*}{$\begin{array}{l}\text { Percentage difference }^{a} \\
\%\end{array}$} \\
\hline & $n$ & Mean & SD & $n$ & Mean & SD & \\
\hline \multicolumn{8}{|c|}{ Fine particulate matter $\left(\mathrm{PM}_{2.5}\right)\left(\mu \mathrm{g} / \mathrm{m}^{3}\right)$} \\
\hline 1-day average & 689 & 17.2 & 9.2 & 690 & 17.0 & 9.1 & 1.0 \\
\hline 7-day average & 695 & 17.9 & 6.1 & 695 & 17.7 & 5.7 & 1.4 \\
\hline 1-month average & 695 & 18.2 & 4.0 & 695 & 18.0 & 3.5 & 1.2 \\
\hline 3-month average & 695 & 18.4 & 3.0 & 695 & 18.1 & 2.4 & 1.4 \\
\hline \multicolumn{8}{|c|}{ Suspended particulate matter (SPM) $\left(\mu \mathrm{g} / \mathrm{m}^{3}\right)$} \\
\hline 1-day average & 682 & 23.0 & 12.0 & 689 & 21.6 & 11.9 & 6.6 \\
\hline 7-day average & 690 & 24.2 & 8.4 & 695 & 22.7 & 7.9 & 6.7 \\
\hline 1-month average & 693 & 24.5 & 5.7 & 695 & 23.1 & 5.0 & 6.0 \\
\hline 3-month average & 695 & 24.7 & 4.8 & 695 & 23.2 & 3.8 & 6.8 \\
\hline \multicolumn{8}{|c|}{ Coarse particulate matter $\left(\mu \mathrm{g} / \mathrm{m}^{3}\right)^{\mathrm{b}}$} \\
\hline 1-day average & 676 & 6.0 & 5.2 & 684 & 4.8 & 5.0 & 24.3 \\
\hline 7-day average & 690 & 6.3 & 4.3 & 695 & 5.0 & 3.7 & 25.4 \\
\hline 1-month average & 693 & 6.3 & 3.4 & 695 & 5.1 & 2.5 & 23.2 \\
\hline 3-month average & 695 & 6.4 & 3.2 & 695 & 5.1 & 1.8 & 26.1 \\
\hline \multicolumn{8}{|c|}{ Nitrogen dioxide $\left(\mathrm{NO}_{2}\right)(\mathrm{ppb})$} \\
\hline 1-day average & 685 & 11.6 & 6.7 & 684 & 14.0 & 7.6 & -17.6 \\
\hline 7-day average & 689 & 11.7 & 5.4 & 695 & 14.0 & 5.3 & -16.9 \\
\hline 1-month average & 692 & 11.8 & 4.8 & 695 & 14.3 & 4.3 & -17.5 \\
\hline 3-month average & 692 & 11.6 & 4.3 & 695 & 14.4 & 3.7 & -19.1 \\
\hline \multicolumn{8}{|c|}{ Photochemical oxidants (Ox) (ppb) } \\
\hline 1-day average & 687 & 44.0 & 15.3 & 695 & 42.8 & 14.8 & 2.8 \\
\hline 7-day average & 688 & 44.5 & 11.7 & 695 & 43.4 & 10.9 & 2.5 \\
\hline 1-month average & 689 & 44.5 & 10.5 & 695 & 43.6 & 9.4 & 2.2 \\
\hline 3-month average & 693 & 44.3 & 9.1 & 695 & 43.5 & 7.9 & 1.9 \\
\hline \multicolumn{8}{|c|}{ Sulphur dioxide $\left(\mathrm{SO}_{2}\right)(\mathrm{ppb})$} \\
\hline 1-day average & 387 & 2.1 & 1.6 & 654 & 1.9 & 1.4 & 12.9 \\
\hline 7-day average & 401 & 2.2 & 1.2 & 678 & 1.9 & 0.8 & 15.3 \\
\hline 1-month average & 408 & 2.3 & 1.1 & 695 & 2.0 & 0.7 & 14.2 \\
\hline 3-month average & 408 & 2.4 & 1.0 & 695 & 2.2 & 0.7 & 11.3 \\
\hline
\end{tabular}

SD standard deviation

${ }^{a}$ (Concentrations measured at the nearest monitoring stations to the respective residential regions - concentrations measured at the Yoshizuka station) $\times 100 /$ concentrations measured at the Yoshizuka station

${ }^{\mathrm{b}}$ Concentrations of coarse particulate matter were defined as the result of subtracting the $\mathrm{PM}_{2.5}$ from the SPM concentrations

the pollutant concentrations measured at the nearest monitoring station to Kyushu University Hospital with those measured at the nearest monitoring stations to the respective residential regions. Hospital-based and residence-based concentrations of $\mathrm{PM}_{2.5}$ and Ox were similar, and the percentage differences of hospital-based and residence-based SPM concentrations were around $6.5 \%$.

As expected, most pregnant women lived a short distance from their delivery hospital, with $40 \%$ of women residing near the Yoshizuka monitoring station, which was the nearest station to Kyushu University Hospital in Fukuoka city, and $84 \%$ of women being assigned pollutant concentrations measured either at the Yoshizuka station or at one of the other four monitoring stations in Fukuoka city. Among women aged 15-49 years who resided in Fukuoka Prefecture, roughly 35\% resided in Fukuoka city [14], a relatively low proportion. Since Kyushu University Hospital is an advanced treatment hospital, the proportion of women with high-risk pregnancies delivering at Kyushu University Hospital is likely to be 
higher than in the local obstetric health care providers. Naturally, not all women with high-risk pregnancies would reside near Kyushu University Hospital. However, the majority of women who delivered at Kyushu University Hospital resided not far from the hospital. With regard to women with low-risk pregnancies in Fukuoka Prefecture, more than $90 \%$ seemed to give birth at the local obstetric health care providers within $30 \mathrm{~min}$ of their home after consideration of satogaeri case [10]. In general, the assumption that pregnant women reside near their delivery hospital is applicable.

Reflecting the strong correlation of $\mathrm{PM}_{2.5}$, SPM, and Ox concentrations among monitoring stations in and outside Fukuoka city, we observed that the $\mathrm{PM}_{2.5}, \mathrm{SPM}$, and $\mathrm{Ox}$ concentrations at the Yoshizuka station were comparable to those at the nearest stations to the residential postal code regions in spite of exposure estimate periods. These results did not contradict the earlier findings that the spatial distribution of fine particles and oxidants was relatively uniform [15]. Since the spatial variation of coarse particles was larger than that of fine particles [16], the correlation coefficients for coarse PM among monitoring stations were not good. In the case of $\mathrm{NO}_{2}$ and $\mathrm{SO}_{2}$, the correlation of concentrations among monitoring stations suggested spatial variation in $\mathrm{NO}_{2}$ and $\mathrm{SO}_{2}$. In particular, the spatial heterogeneity of $\mathrm{NO}_{2}$, due to its multiple emission sources, is well known [17]. Since the same measurement method for each of $\mathrm{NO}_{2}$ and $\mathrm{SO}_{2}$ was used in the monitoring stations within Fukuoka city, the influence of the measurement method to the comparison between hospital-based and residencebased concentrations was likely to be small. Therefore, exposure estimations for coarse $\mathrm{PM}, \mathrm{NO}_{2}$, and $\mathrm{SO}_{2}$ based on the delivery hospital must be more tentative than those for $\mathrm{PM}_{2.5}$, SPM, and Ox.

The present study has a number of limitations. We estimated pollutant exposure at each woman's home using pollutant concentrations measured at the nearest monitoring stations to their respective residential postal code region. Although the majority of women resided within a $5-\mathrm{km}$ radius of their respective monitoring station, our estimation still may not accurately reflect the pollutant concentrations at their homes due to spatial variation in pollutant concentrations within the region where a monitoring station covers $[18,19]$. Furthermore, some women resided in areas far from Kyushu University Hospital, yet some of these women may have returned to parental homes nearer to this hospital, and thereby given birth at the hospital. Although we excluded women who resided outside Fukuoka Prefecture under the assumption of the satogaeri case, we did not know of any satogaeri case in the Prefecture. If we had excluded such satogaeri cases, the accuracy of the assumption that pregnant women resided near their delivery hospital may have been more clearly determined. Finally, we did not consider maternal time-activity pattern. There is a report in Canada that time-active pattern does not have a large impact on exposure estimation for pregnant women who spend a lot of time at home [20], though this may not be applied for the Japanese pregnant women.

In conclusion, our hypothesis that pollutant exposure estimation based on the delivery hospital approximates that based on the home address of pregnant women is likely to be acceptable for $\mathrm{PM}_{2.5}$, SPM, and Ox.

\section{Additional file}

Additional file 1: Table S1. Pearsons' correlation coefficients for daily mean pollutant concentrations among monitoring stations in Fukuoka, 2014. Table S2. Pearson's correlation coefficients between pollutant concentrations measured at the Yoshizuka monitoring station and those at the nearest monitoring stations to the respective residential postal code regions. (XLSX $20 \mathrm{~kb}$ )

\section{Abbreviations}

$\mathrm{NO}_{2}$ : Nitrogen dioxide; Ox: Photochemical oxidants; PM: Particulate matter; $\mathrm{PM}_{2.5}$ : Fine particulate matter; $\mathrm{SO}_{2}$ : Sulphur dioxide; SPM: Suspended particulate matter

\section{Acknowledgements}

We thank Ms. Noriko Shidai of the Japan National Institute for Environmental Studies for her assistance in this study.

\section{Funding}

This research received no specific grant from any funding agency in the public, commercial, or non-profit sectors.

\section{Availability of data and materials}

The dataset used and analysed during the current study is available from the corresponding author on reasonable request.

\section{Authors' contributions}

TM conceptualised and designed the study, collected the data, analysed the data, interpreted the results, and drafted the manuscript. SM designed the study, collected the data, analysed the data, interpreted the results, and assisted with the preparation of the manuscript. HN and KK participated in the literature review and interpretation of the results and revised the manuscript. SY participated in the study design, statistical analysis, and review of the results and manuscript. All authors read and approved the final manuscript.

\section{Competing interests}

The authors declare that they have no competing interests.

Consent for publication

Not applicable.

\section{Ethics approval and consent to participate}

The study protocol was approved by the Institutional Review Board of Kyushu University, Japan, and the Japan National Institute for Environmental Studies. We collected aggregated data, so individual informed consent was not required.

\section{Publisher's Note}

Springer Nature remains neutral with regard to jurisdictional claims in published maps and institutional affiliations.

\section{Author details}

${ }^{1}$ Environmental Epidemiology Section, Centre for Health and Environmental Risk Research, National Institute for Environmental Studies, 16-2 Onogawa, Tsukuba, Ibaraki 305-8506, Japan. ${ }^{2}$ Department of Obstetrics and 
Gynaecology, Kyushu University Hospital, Kyushu University, 3-1-1 Maidashi, Higashi-ku, Fukuoka 812-8582, Japan.

Received: 5 March 2017 Accepted: 4 June 2017

Published online: 13 June 2017

\section{References}

1. Ha EH, Hong YC, Lee BE, Woo BH, Schwartz J, Christiani DC. Is air pollution a risk factor for low birth weight in Seoul? Epidemiology. 2001;12:643-8.

2. Bell ML, Ebisu K, Belanger K. Ambient air pollution and low birth weight in Connecticut and Massachusetts. Environ Health Perspect. 2007;115:1118-24.

3. Stieb DM, Chen L, Hystad P, Beckerman BS, Jerrett M, Tjepkema M, et al. A national study of the association between traffic-related air pollution and adverse pregnancy outcomes in Canada, 1999-2008. Environ Res. 2016:148:513-26.

4. Wilhelm M, Ritz B. Local variations in $\mathrm{CO}$ and particulate air pollution and adverse birth outcomes in Los Angeles County, California, USA. Environ Health Perspect. 2005;113:1212-21.

5. Malmqvist E, Rignell-Hydbom A, Tinnerberg H, Bjõrk J, Stroh E, Jakobsson K, et al. Maternal exposure to air pollution and birth outcomes. Environ Health Perspect. 2011;119:553-8.

6. Savitz DA, Bobb JF, Carr JL, Clougherty JE, Dominici F, Elston B, et al. Ambient fine particulate matter, nitrogen dioxide, and term birth weight in New York, New York. Am J Epidemiol. 2014;179:457-66.

7. Le HQ, Batterman SA, Wirth JJ, Wahl RL, Hoggatt KJ, Sadeghnejad A, et al. Air pollutant exposure and preterm and term small-for-gestational-age births in Detroit, Michigan: long-term trends and associations. Environ Int. 2012;44:7-17.

8. Ministry of Education, Culture, Sports, Science and Technology, and Ministry of Health, Labour and Welfare, Japan. Ethical guidelines for medical and health research involving human subjects (as of 28 Feb 2017). http://www. mhlw.go.jp/file/06-Seisakujouhou-10600000-Daijinkanboukouseikagakuka/ 0000153339.pdf. Accessed 17 Apr 2017.

9. Inui T, Hayashi I, Inomata R. Approximate time required associated reasons for selection and change birthing facility. Nihonboshikangogakukaishi. 2015;8:53-8 (in Japanese).

10. Nakashima K, Hirata N, Fukushima K. Medical facility selection behavior of low-risk pregnancy women. Kurumeigakukaizashi. 2008;71:369-75 (in Japanese with English abstract).

11. Kloog I, Melly SJ, Coull BA, Nordio F, Schwartz JD. Using satellite-based spatiotemporal resolved air temperature exposure to study the association between ambient air temperature and birth outcomes in Massachusetts. Environ Health Perspect. 2015:123:1053-8.

12. Rich DQ, Liu K, Zhang J, Thurston SW, Stevens TP, Pan Y, et al. Differences in birth weight associated with the 2008 Beijing Olympics air pollution reduction: results from a natural experiment. Environ Health Perspect. 2015;123:880-7.

13. Lee SJ, Hajat S, Steer PJ, Filippi V. A time-series analysis of any short-term effects of meteorological and air pollution factors on preterm births in London, UK. Environ Res. 2008;106:185-94.

14. Statistics Bureau, Ministry of Internal Affairs and Communications, Japan. 2015 population census. http://www.stat.go.jp/english/data/kokusei/index. htm. Accessed 17 Apr 2017.

15. Sarnat SE, Klein M, Sarnat JA, Flanders WD, Waller LA, Mulholland JA, et al. An examination of exposure measurement error from air pollutant spatial variability in time-series studies. J Exp Sci Environ Epidemiol. 2010;20:135-46.

16. Liu Y-J, Harrison RM. Properties of coarse particles in the atmosphere of the United Kingdom. Atmos Environ. 2011;45:3267-76.

17. Yamaguchi $H$, Takashima H, Maruyama Y. Spatiotemporal inhomogeneity in nitrogen dioxide $\left(\mathrm{NO}_{2}\right)$ over Fukuoka observed by Car MAX-DOAS. J Jpn Soc Atmos Environ. 2016;51:238-44 (in Japanese with English abstract).

18. Michikawa T, Nakai S, Nitta H, Tamura K. Validity of using annual mean particulate matter concentrations as measured at fixed site in assessing personal exposure: an exposure assessment study in Japan. Sci Total Environ. 2014:466-467:673-80.

19. Monn C. Exposure assessment of air pollutants: a review on spatial heterogeneity and indoor/outdoor/personal exposure to suspended particulate matter, nitrogen dioxide and ozone. Atmos Environ. 2001;35:1-32

20. Nethery E, Leckie SE, Teschke K, Brauer M. From measures to models: an evaluation of air pollution exposure assessment for epidemiological studies of pregnant women. Occup Environ Med. 2008;65:579-86.

\section{Submit your next manuscript to BioMed Central and we will help you at every step:}

- We accept pre-submission inquiries

- Our selector tool helps you to find the most relevant journal

- We provide round the clock customer support

- Convenient online submission

- Thorough peer review

- Inclusion in PubMed and all major indexing services

- Maximum visibility for your research

Submit your manuscript at www.biomedcentral.com/submit
) Biomed Central 